\title{
Computational study on non-asymptotic behavior of developing turbulent pipe flow
}

\author{
W. A. S. Kumara ${ }^{1}$, B. M. Halvorsen ${ }^{1,2} \&$ M. C. Melaaen ${ }^{1,2}$ \\ ${ }^{I}$ Telemark University College, Norway \\ ${ }^{2}$ Telemark $R \& D$ Centre (Tel-Tek), Norway
}

\begin{abstract}
In general, developing turbulent pipe flow is a transition from a boundary layer type flow at the entrance to a fully developed flow downstream. The boundary layer thickness grows as the distance from the pipe inlet increases. An accurate description of the velocity and pressure distribution within the entrance region is very important to calculate the pressure drop for hydrodynamic inlets. More important perhaps, the velocity distribution is needed for an analysis of forced convection and mass transfer in a tube entrance. In the current study, we report the results of a detailed and systematic numerical investigation of developing turbulent pipe flow. Two-dimensional, axisymmetric computational scheme has been devised for determining the flow development in the entrance region of a circular pipe at different Reynolds numbers. The simulations are performed using commercial CFD software ANSYS FLUENT 12.0. Non-asymptotic behavior observed in developing turbulent pipe flow is discussed in detail. The predicted results are also compared with literature data.
\end{abstract}

Keywords: non-asymptotic behavior, turbulent flow, laminar flow, fully developed flow, computational simulations, axial velocity, velocity overshoot, wall shear stress.

\section{Introduction}

Turbulent pipe flow is one of the most common fluid motion in industrial and engineering applications. The importance of knowing the length of pipe required for the turbulent flow to fully develop, i.e., the velocity profile to become nonvarying in the axial direction, has long been recognized. Consequently, several investigators have attempted to obtain solutions for incompressible turbulent 
flow development in pipes or ducts using numerical techniques [1-4]. The flow in the inlet region of a pipe is a transition from a boundary layer flow at the entrance to a fully developed flow downstream. Until recently, it is expected that the flow near the entrance of a pipe should resemble boundary layer flow over a flat plate [5]. Therefore, the peakiness of the velocity profile, defined as the ratio between the maximum value and the spatial mean, was thought to increase gradually until the boundary layers meet at the pipe centreline. According to the measurements quoted by Schlichting [6], this appeared to occur in turbulent flow after 25 to 100 pipe diameters, the exact length depending on the Reynolds number. Thereafter the flow was considered fully developed. In fact, however developing turbulent pipe flow is much more complex and there is a significant difference between the behavior of the free stream in flow over a plate and in a pipe [7]. Unlike that encountered in flat-plate boundary layer flow, the free stream in the inlet region of a pipe is completely surrounded by a growing boundary layer. Thus, the free stream accelerates as the displacement thickness of the boundary layer grows, and it loses its identity downstream as the boundary layer thickness reaches a value equal to the pipe radius. Following the disappearance of the free stream, further changes in the velocity profile and turbulence structure occur before a fully developed condition (i.e. uniform flow) is reached [7]. Bradley (quoted in [8]) appears to be the first who discovered that velocity profile peakiness might reach a maximum, which he found at about 40 pipe diameters, and then decreased again. Weir et al. [9] also obtained a maximum core velocity at 40 pipe diameters, while gradual velocity increase along the pipe were measured by Deissler [10] and Sale [11]. The latter type of velocity distribution was produced in [9] also, when a trip ring was inserted at the pipe entrance. Thus, it was shown that a change of inlet conditions might create substantial differences in flow development. Reichert and Azad presented detailed measurements of axial mean velocity profiles, in the inlet 70 diameters of the pipe, showing that the development of turbulent pipe flow is nonasymptotic [12]. The experiments were done at seven Reynolds numbers in the range 56 000-153 000. An axial velocity peak exceeding the fully developed values has been found to occur along the pipe centreline. Hot film measurements of the mean wall shear stresses in the inlet region also show a non-asymptotic development consistent with the mean velocity results. The Reynolds number behaviour of the peak position has been determined. They suggested that the peak position is a function of Reynolds number given by:

$$
\frac{x_{p}}{D}=30.80+\frac{0.89}{10^{-5} \mathrm{Re}}+\frac{0.79}{\left(10^{-5} \mathrm{Re}\right)^{2}}
$$

where $\mathrm{x}_{\mathrm{p}}$ is the axial distance to the peak position, $\mathrm{D}$ is the pipe diameter and $\mathrm{Re}$ is Reynolds number. This equation implied a limit of $\mathrm{x} / \mathrm{D} \sim 31$ for the peak position at the highest incompressible Reynolds numbers. On the other hand, the analysis implied that rather long entrance length would be required for low Reynolds number flows to become fully developed. Klein [5] has given a review of experimental results on velocity profile development in turbulent pipe flows. 
In the current study, we report the results of a detailed and systematic numerical investigation of developing turbulent pipe flow. The present results also indicate that the development of the velocity profile to its fully developed value is not monotonic but displays a pronounced overshoot and consequently the fully developed state is not reached until some distance downstream. Since such flows are frequently used to provide reference or control inlet conditions for general flow investigations it is important that they should be better understood.

\section{Turbulent pipe flow}

In general, the major velocity changes take place very close to the body surface [13]. Nevertheless, there is generally some ambiguity in speaking of the "boundary layer thickness" if what is meant in the region in which velocity changes from zero to free stream velocity. It is convenient and far more useful to define boundary layer thickness parameters that are completely unambiguous [13]. They are called the displacement thickness $\left(\delta_{1}\right)$ and the momentum thickness $\left(\delta_{2}\right)$. The displacement thickness is defined as follows:

$$
\delta_{1}=R \int_{0}^{1}\left(1-\frac{u}{u_{c}}\right) \frac{r}{R} d\left(\frac{r}{R}\right)
$$

where $R$ is the pipe radius, $u$ is the mean axial velocity, $u_{c}$ is the centreline velocity and $\mathrm{r}$ represents the radial location in the pipe. The momentum thickness is defined as:

$$
\delta_{2}=R \int_{0}^{1} \frac{u}{u_{c}}\left(1-\frac{u}{u_{c}}\right) \frac{r}{R} d\left(\frac{r}{R}\right)
$$

The measure of the flow development used in this paper is Sovran and Klomp's [14] blockage factor (b) which is defined by the ratio of the axisymmetric displacement thickness $\left(\delta_{1}\right)$ to pipe radius R. It can also be estimated by using bulk velocity $\left(\mathrm{u}_{\mathrm{b}}\right)$ and centreline velocity $\left(\mathrm{u}_{\mathrm{c}}\right)$ of the flow as given below:

$$
b=2 \frac{\delta_{1}}{R}=1-\frac{u_{b}}{u_{c}}
$$

\section{Computational scheme}

The commercial CFD software package, FLUENT 12.0, which is based on the finite volume approach, is used for solving the set of governing equations. The discretized equations, along with the initial and boundary conditions, are solved using the pressure based solution method to obtain a numerical solution. Using the pressure based solver, the conservation of mass and momentum are solved iteratively and a pressure-correction equation is used to ensure the conservation of momentum and mass. Standard k- $\omega$ model is used for modelling turbulence. In addition, standard $\mathrm{k}-\varepsilon$ model and $\mathrm{RNG} \mathrm{k}-\varepsilon$ model together with enhanced 
wall treatment as the near-wall modelling method is used to investigate the effect of turbulence model on the flow development.

A schematic representation of the computational domain is given in Figure 1. At inlet $(x=0)$, we apply a uniform velocity $u_{b}$. No-slip boundary condition is applied on the pipe wall and the pipe outlet is treated as a pressure outlet. One plane in the axisymmetric pipe is simulated and pipe centreline is considered as the axisymmetry axis. The length of the computational domain is dependent on the Reynolds number of the flow in question. In general, the domain was at least three times as long as the calculated development length.

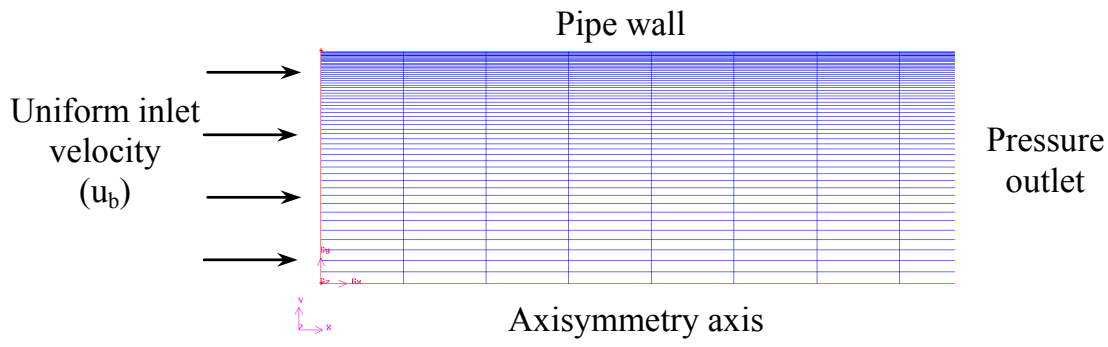

Figure 1: Computational domain.

Two-dimensional, axisymmetric grid system is used for the computations. The assumption of axisymmetry implies that there are no circumferential gradients in the flow. In this case, FLUENT used the cylindrical representation of the incompressible Navier-Stokes equations by taking the advantage of axisymmetry of the flow field. Enhanced wall treatment is a near-wall modeling method that combines a two-layer model with so-called enhanced wall functions [15]. A boundary layer mesh is used close to the wall, which is fine enough to be able to resolve the viscous sublayer (first near-wall node placed at $\mathrm{y}^{+}<5$ ).

\section{Results}

\subsection{Asymptotic behaviour of developing laminar flow}

The development of laminar flow is investigated prior to the study of developing turbulent flows. Laminar flow $(\mathrm{Re}=557)$ in a pipe with internal diameter $56 \mathrm{~mm}$ is simulated and the results are given in Fig. 2. The uniform inlet velocity is $0.01 \mathrm{~m} / \mathrm{s}$ and water is used as the working fluid. Fig. 2(a) shows the fully developed velocity profile at $\mathrm{x} / \mathrm{D}=160$. The velocity profile is parabolic and the maximum axial velocity is $0.02 \mathrm{~m} / \mathrm{s}$. In general, the axial velocity profile of laminar flow in pipes is given by the following expression:

$$
u=2 u_{b}\left(1-\frac{r^{2}}{R^{2}}\right)
$$

Eq. (5) suggests that the maximum axial velocity at the pipe center is $0.02 \mathrm{~m} / \mathrm{s}$ and shows a good agreement with the simulated results. Fig. 2(b) shows the 


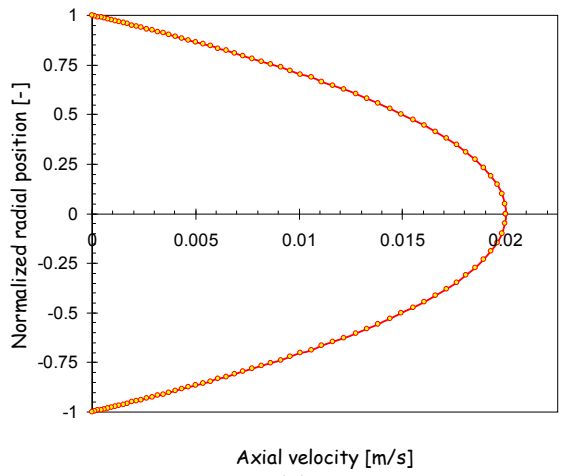

(a)

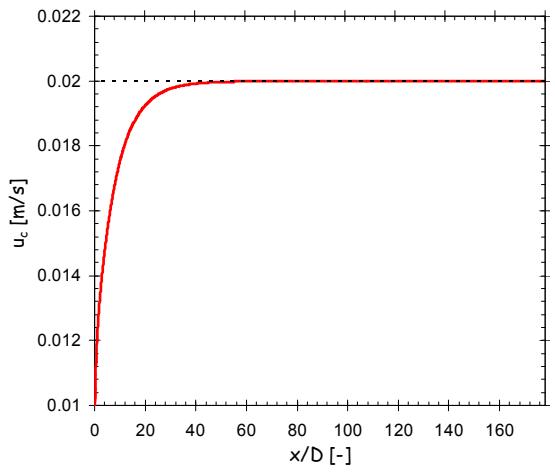

(b)

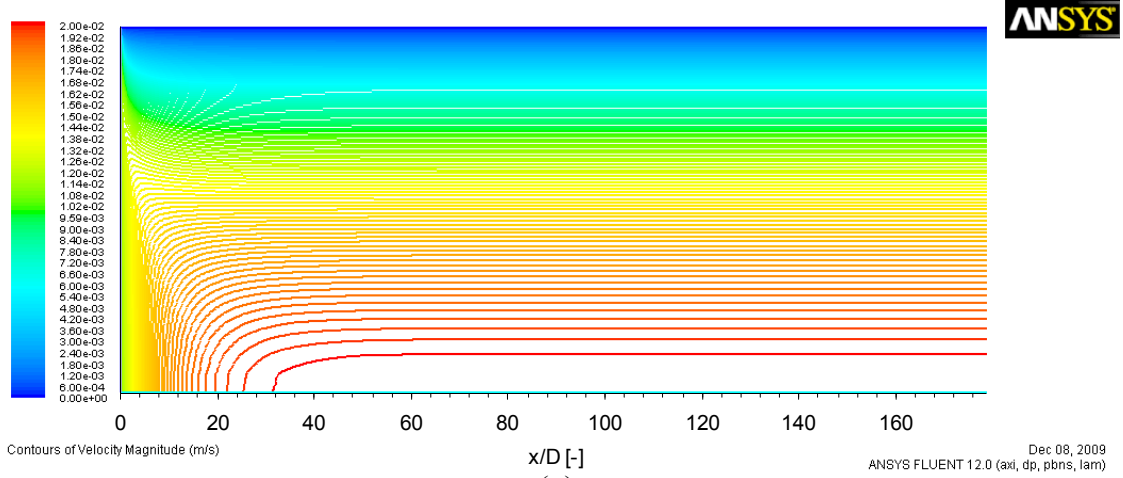

(c)

Figure 2: Asymptotic behavior of developing laminar flow $(\operatorname{Re}=557)$ : (a) Axial velocity, (b) Development of the axial velocity profile with non-dimensional distance from the inlet, (c) Contours of axial velocity.

evolution of the centreline velocity along the pipe. As the fluid moves down the tube, a boundary layer of low-velocity fluid forms and grows on the surface because the fluid immediately adjacent to the surface must have zero velocity. This retardation near the wall spreads inwards owing to viscous effects and the slowed-down fluid close to the wall causes the fluid in the center to move faster, since the cross-sectional mass flow rate at any axial location remains constant. A particular feature of viscous flow inside cylindrical tubes is the fact that the boundary layer must meet itself at the tube centerline, and the velocity distribution then establishes a fixed pattern that is invariant thereafter. The part of the tube in which the momentum boundary layer grows and the velocity distribution changes with length is referred as the hydrodynamic entry length $\left(\mathrm{x}_{\mathrm{d}}\right)$. It is defined as the axial distance required for the centreline velocity to reach $99 \%$ of its fully developed value. As shown in Fig. 2(b), the centreline velocity gradually increases and reaches its fully developed value asymptotically. In this case, the hydrodynamic entry length is $x_{d} / D=31.78$. Durst et al. [16] have 
conducted a detailed numerical study and proposed the following nonlinear correlation for hydrodynamic entry length:

$$
\frac{x_{d}}{D}=\left[0.619^{1.6}+(0.0567 \mathrm{Re})^{1.6}\right]^{1 / 1.6}
$$

The calculated entry length ( $\left.x_{d} / D\right)$ according to Eq. (6) at $R e=557$ is 31.62 . Therefore, the present predictions show a good consensus with the results presented by Durst et al. [16]. Contour plot of mean axial velocity field is given in Fig. 2(c). It clearly shows the asymptotic behavior of developing laminar flow in pipes.

\subsection{Non-asymptotic behaviour of developing turbulent flow}

The development of turbulent pipe flow is investigated in this section. Turbulent flow $(\mathrm{Re}=55776)$ in a pipe with internal diameter $56 \mathrm{~mm}$ is simulated and the results are given in Fig. 3. Water is used as the working fluid. The predictions are based on standard k- $\omega$ model. Fig. 3(a) shows the fully developed axial velocity profile at $x / D=160$. The velocity profile is flat compared to the laminar flow as expected. The maximum mean axial velocity is $1.189 \mathrm{~m} / \mathrm{s}$. The axial velocity profile of turbulent pipe flow is given by the power-law as follows:

$$
\frac{u}{u_{c}}=(1-r / R)^{1 / n}
$$

where $\mathrm{n}$ is the power-law exponent and determined by:

$$
n=-1.7+1.8 \log \mathrm{Re}
$$

The power-law profile is much more blunt than the laminar profile, $\mathrm{u}_{\mathrm{b}} \sim \mathrm{u}_{\mathrm{c}}$, but is more generally determined by:

$$
\frac{u_{b}}{u_{c}}=\frac{2 n^{2}}{(n+1)(2 n+1)}
$$

In this case at $\mathrm{Re}=55776$, Eq. (9) gives $\mathrm{u}_{\mathrm{b}} / \mathrm{u}_{\mathrm{c}}=0.813$, while the simulated results gives $\mathrm{u}_{\mathrm{b}} / \mathrm{u}_{\mathrm{c}}=0.84$.

Developing turbulent pipe flow is basically a transition from a boundary layer type flow at the entrance to a fully developed flow downstream. The boundary layer thickness grows as the distance from the pipe inlet increases. In order to maintain a constant bulk flow rate, the core region accelerates as it is being squeezed by the growing boundary layer. In the initial portion of the entrance flow, the boundary layers serve to squeeze fluid into the core causing the core velocity to increase. This core acceleration is the combined result of the deceleration near the wall and conservation of mass. As the flow evolves, the boundary layer grows and eventually merges. The flow subsequently evolves to its fully developed state. The initial core acceleration is so large for a turbulent flow that the centreline velocity overshoots its fully developed value as shown in Fig. 3(b). Hence, the development of turbulent pipe flow shows non-asymptotic behavior in contrast with the results of laminar flow. This velocity overshoot is more evident on the contour plot of axial velocity as shown in the Fig. 3(c). The 


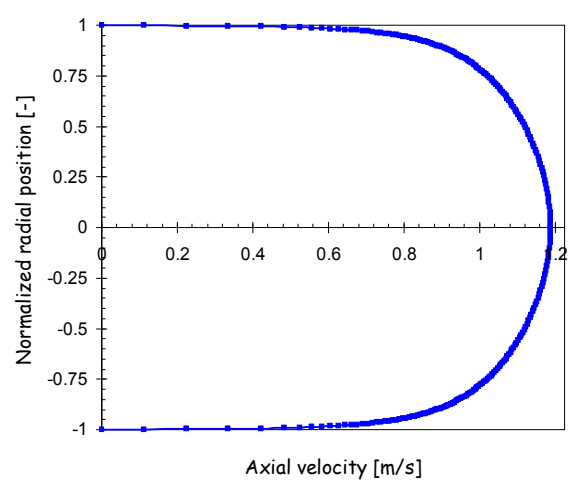

(a)

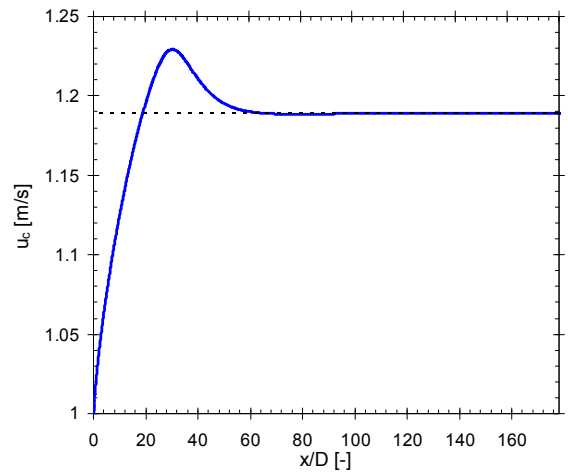

(b)

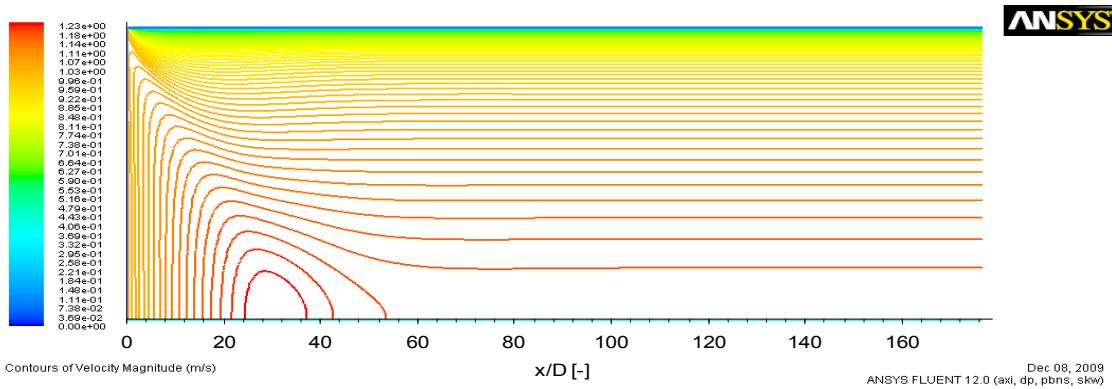

(c)

Figure 3: Non-asymptotic behavior of developing turbulent flow $(\operatorname{Re}=$ 55776): (a) Axial velocity, (b) Development of the axial velocity profile with non-dimensional distance from the inlet, (c) Contours of axial velocity.

centreline velocity increases up to about 30 pipe diameters, and followed by a decrease up to about 65 diameters, and then attains an approximately constant value. However, there is a very small undershoot of the centreline velocity profile compared to the fully developed value after the peak velocity. This waviness is due to the adjustment of the shear stress in the central region, an extremely complicated process since shear stresses of any direction exist [17]. The axial velocity contour plots are characterized by a narrowing core of accelerating fluid, which disappears after 24-37 pipe diameters. Outer regions are characterized by decelerating axial flow over approximately the first 20 diameters. This leads to axial velocities for radial positions near the wall, which undershoot the final fully developed values. The peak position of the centreline velocity is observed at $\mathrm{x}_{\mathrm{p}} / \mathrm{D}=31.25$. The same position can be calculated by using Eq. (1) and it gives $x_{p} / D=34.93$ [18]. The reason that causes this discrepancy is uncertain. However, the differences of the inlet conditions used for experiments and simulations can influence the position of the centreline velocity overshoot. 


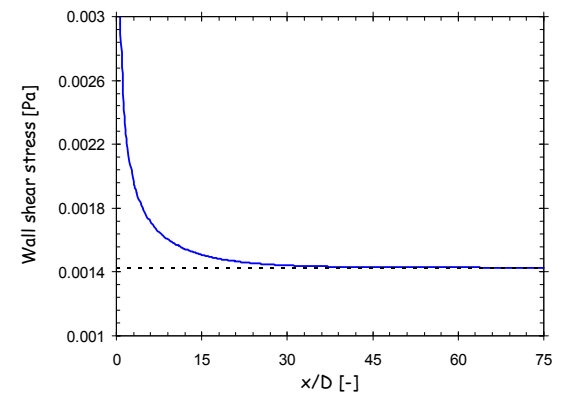

(a)

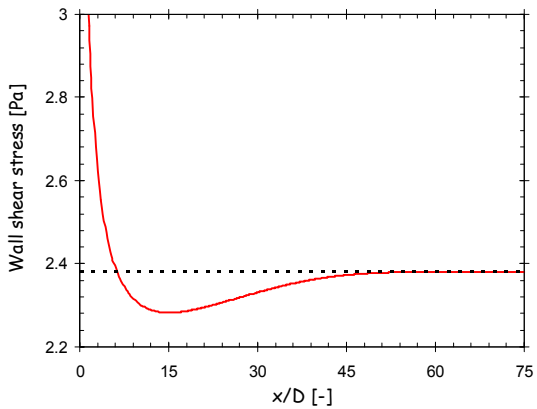

(b)

Figure 4: Wall shear stress: (a) Laminar flow $(\mathrm{Re}=557)$, (b) Turbulent flow $(\mathrm{Re}=55776)$.

Fig. 4 shows the wall shear stress distribution for developing laminar and turbulent flows. At the entrance of the pipe, there are larger shear rates by the wall giving higher wall shear stresses. As the boundary layer grows, shear rates close to the wall decreases. When the flow is fully developed, shear rates do not change along the pipe and constant wall shear stress is observed. As shown in Fig. 4(a), the wall shear stress decreases monotonically and attains its fully developed value for laminar flow. Fig. 4(b) shows wall shear stress distribution of developing turbulent flow. Significantly higher wall shear stresses are observed in turbulent flow compared to the laminar flow. The wall shear stress decreases as the boundary layer grows along the pipeline. However, wall shear stress undershoots its fully developed value compared to the laminar flow. It decreases up to 15 pipe diameters and then gradually increases up to 57 pipe diameters to reach its fully developed value. This may be attributed to the undershoot of the axial velocity gradient close to the wall around the position of peak centreline velocity.

\subsection{Reynolds number effect on centerline velocity overshoot}

In this section, the effect of Reynolds number on the position of centreline velocity overshoot is investigated. Fig. 5 shows contour plots of mean axial velocity at two different Reynolds numbers corresponding to the flows at bulk velocities $0.25 \mathrm{~m} / \mathrm{s}$ and $1.50 \mathrm{~m} / \mathrm{s}$, respectively. Comparison of the axial velocity contour plots showed that the centreline velocity peak is more pronounced for the lowest Reynolds number. The maximum value of $u_{p} / u_{b}$ approached 1.23 for $\mathrm{Re}=13994$, while for $\mathrm{Re}=83664$ the maximum $\mathrm{u}_{\mathrm{p}} / \mathrm{u}_{\mathrm{b}}$ value just exceed 1.18 .

An analysis of centreline velocities was undertaken to determine the effect of Reynolds number on the velocity overshoot position. Fig. 6 shows the flow development at seven different Reynolds numbers. The calculated blockage factor is plotted against the non-dimensional pipe length. The centerline peak overshoot position slightly moves downstream as the Reynolds number increases. Larger Reynolds numbers are seen to produce lower peak values and smaller Reynolds numbers higher peak values. The present predictions show a 

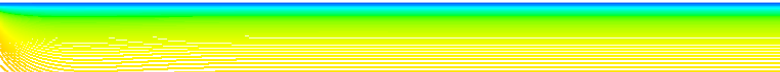

\section{MNYS}

Contours of Velocity Magnitude (mis)
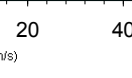

60

80

$\mathrm{x} / \mathrm{D}[-]$

(a)

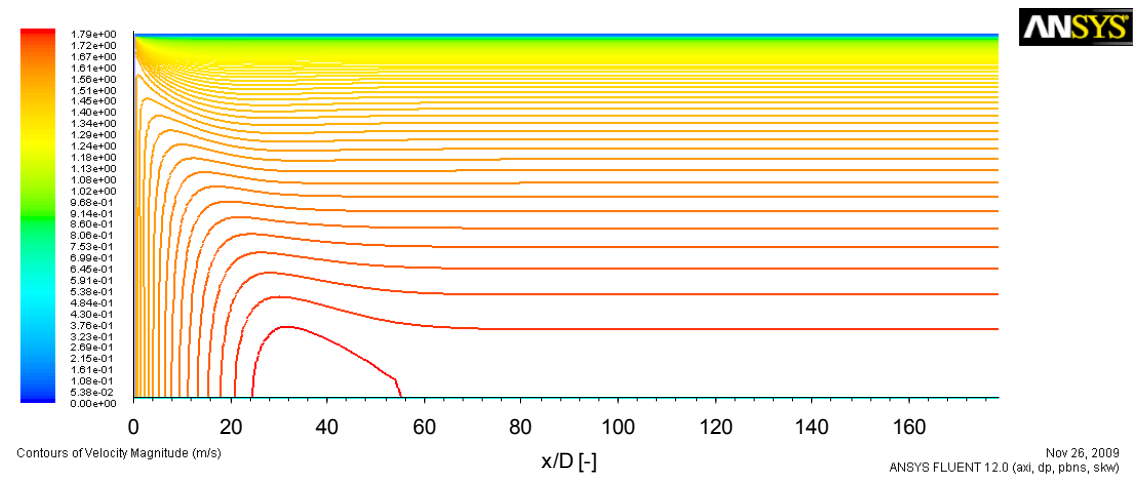

(b)

Figure 5: Contours of axial velocity: (a) $U=0.25 \mathrm{~m} / \mathrm{s}(\mathrm{Re}=13994)$, (b) $\mathrm{U}=$ $1.50 \mathrm{~m} / \mathrm{s}(\operatorname{Re}=83664)$.

good agreement with the experimental results presented by Klein [5]. The Reynolds number effect on the centerline peak position can be explained by considering integral boundary layer parameters. Axial displacement and momentum thicknesses (normalized by pipe diameter) have been computed by numerical integration of the axial velocity profiles and presented in Fig. 7. The behavior of these integral boundary layer parameters is significant in developing turbulent flow because the parameters are related to the momentum flux terms appearing in the boundary layer momentum balance equation [18]. As shown in the figure longitudinal derivatives of these integral parameters do not become zero monotonically providing a clear evidence of non-asymptotic character of the 


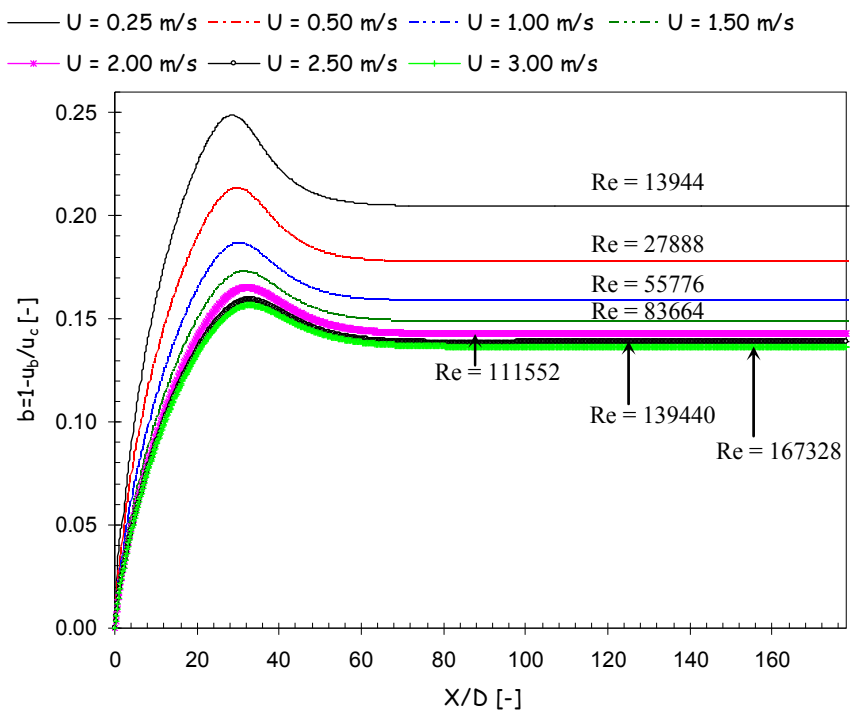

Figure 6: Development of the axial velocity profile with non-dimensional distance from inlet.

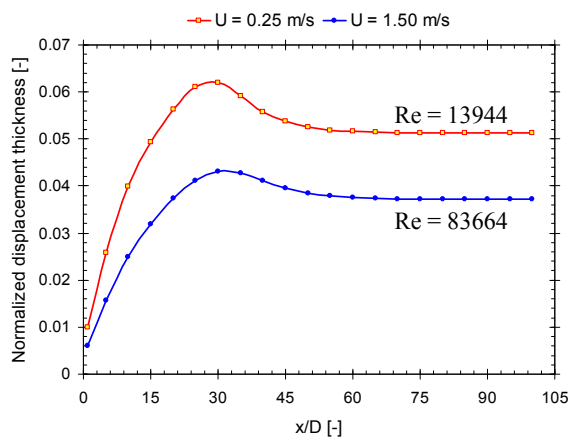

(a)

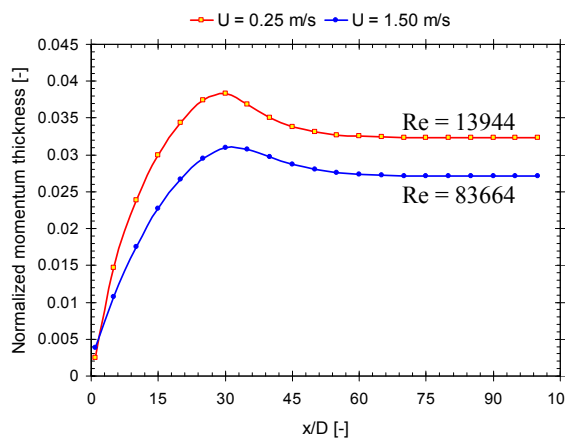

(b)

Figure 7: Normalized displacement and momentum thicknesses: (a) displacement thickness, (b) momentum thickness.

flow development. Both displacement and momentum thickness curves show a distinct peak in their development along the pipe. The results show that more rapid boundary layer growth occurs for the lower Reynolds number since thicknesses for the lower Reynolds number are largest. This is the usual trend observed for turbulent boundary layer growth in other flows such as on a flat plate. As a result of rapid development of the boundary layer at low Reynolds numbers, centerline velocity overshoot can be observed close to the pipe entrance. As the Reynolds number increases the peaks of the displacement and 


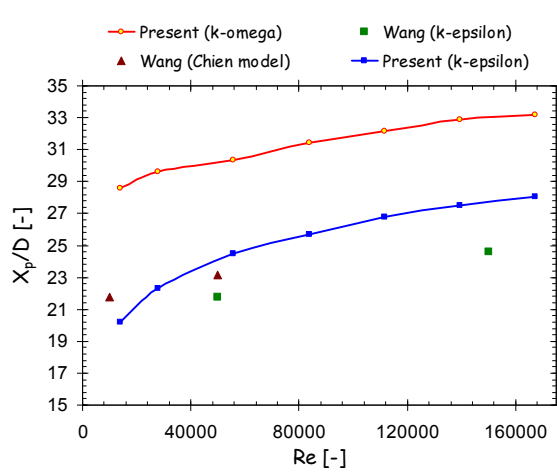

(a)

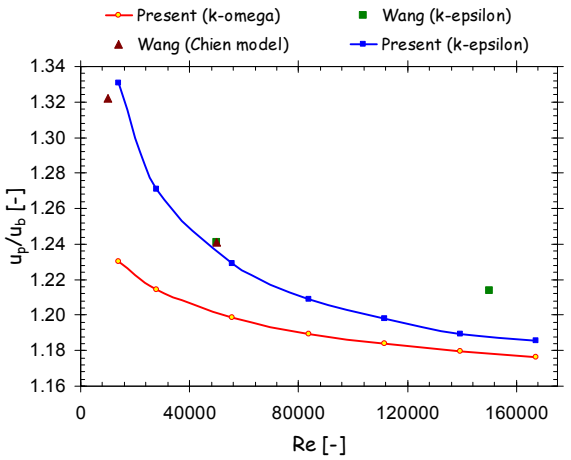

(b)

Figure 8: Overshoot of the axial velocity profile in developing turbulent flow: (a) $\mathrm{x}_{\mathrm{p}} / \mathrm{D}$, (b) $\mathrm{u}_{\mathrm{p}} / \mathrm{u}_{\mathrm{b}}$.

momentum thickness curves moves towards the downstream. Therefore, the position where the centerline velocity overshoot is observed moves downstream as the Reynolds number increases. It should be mentioned that a discrepancy has been found between our observation and Reichert's [18] experimental data regarding the effect of Reynolds number on the location of the centerline overshoot position. Reichert suggested that the peak position is a function of Reynolds number given by Eq. (1) [18]. That means the peak overshoot position decreases as Reynolds number increases. The reason that causes this discrepancy is uncertain. However, the present predictions show a good agreement with the results presented by Wang [4] as shown in Fig. 8. Wang has reported that the peak overshoot position moves downstream as the Reynolds number increases. He has used standard k- $\varepsilon$ model and Chien model [19] for turbulence modelling. The present predictions of centerline velocity overshoot position using standard $\mathrm{k}-\varepsilon$ model agree well with the results presented by Wang using same model as shown in Fig. 7(a). The magnitude of the velocity overshoot $\left(\mathrm{u}_{\mathrm{p}} / \mathrm{u}_{\mathrm{b}}\right)$ decreases as the Reynolds number increases. The present predictions and the results presented by Wang using standard k- $\varepsilon$ model show good consensus as shown in Fig. 7(b). The predicted behavior of the centerline velocity overshoot based on standard $\mathrm{k}$ $\omega$ is also given in Fig. 7. There are significant deviations between the predictions based on standard k- $\varepsilon$ and standard k- $\omega$ models. However, the predicted results show some similarities in their overall shape. The reason that causes this discrepancy is uncertain. Flow turbulence plays an important role in the physical process involved in the non-asymptotic mean flow development [18]. This suggested that the non-asymptotic behavior could be characteristic of both the mean velocity and turbulence stresses. These models handle turbulent quantities in different ways and it can be the reason for the observed deviations. A further investigation is needed in order to understand the effect of turbulence on the nonasymptotic turbulent flow development. 


\subsection{Comparison of predicted results with experimental data}

The present predictions are compared with the experimental data presented by Reichert [18]. The wind tunnel installation used for the experiments has been described in detail by Reichert [18]. Briefly, air was blown through an 89:1 contraction cone, tripped by a strip of sandpaper $(50.8 \mathrm{~mm})$, and allowed to develop along a 76 diameter length of $101.6 \mathrm{~mm}$ inside diameter steel pipe, before being exhausted through an $8^{\circ}$ included angle conical diffuser. Profiles of the axial component of the mean velocity were calculated using dynamic pressures, which were obtained from differential pressure measurements between a traversing total pressure tube and a ring of static pressure taps in the same plane. A Betz projection manometer with $0.1 \mathrm{~mm}$ of water scale intervals was used. No corrections were attempted to account for turbulence or displacement effects on the pressure tube. Present simulations are based on the RNG k- $\varepsilon$ turbulence model. The simulated case has the flowing physical conditions: $\mathrm{u}_{\mathrm{b}}=17.3 \mathrm{~m} / \mathrm{s}$, density $=0.118 \mathrm{~kg} / \mathrm{m}^{3}$, kinematic viscosity $=1.59 \times 10^{-5} \mathrm{~m}^{2} / \mathrm{sec}$.

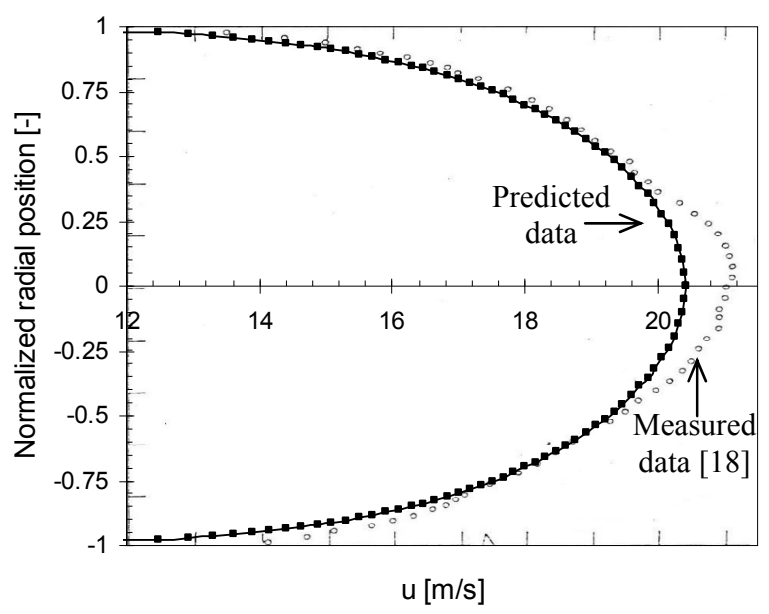

Figure 9: Comparison of mean axial velocity $(\mathrm{x} / \mathrm{D}=61.9)$.

Fig. 8 gives a comparison of mean axial velocity profiles. A good comparison is observed between measurements [18] and predictions of axial velocity. However, there are some deviations close to the pipe center. The maximum deviation observed is about $1.45 \%$. Fig. 9 shows a comparison of contour plots of axial velocity. It is notable that the simulation can predict the measured [18] behavior of the centerline velocity overshoot. However, there are some discrepancies. The measured maximum value of $\mathrm{u} / \mathrm{u}_{\mathrm{b}}$ approaches to 1.27 . The predicted value approaches to 1.19 . Hence, the experiments produce a larger velocity overshoot compared to the predicted data. The measured results shows that the centerline velocity overshoot is located around $34<x / D<35.5$. However, the predicted data shows the corresponding location around $25<\mathrm{x} / \mathrm{D}<31.5$. Hence, the predicted velocity overshoot position is located closer to the pipe inlet 


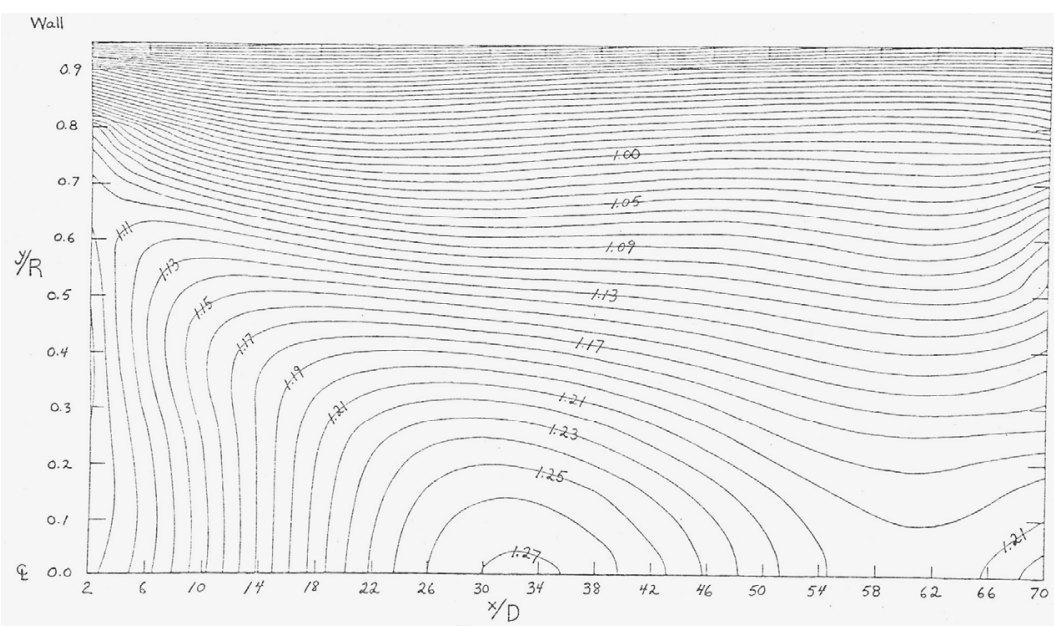

(a)

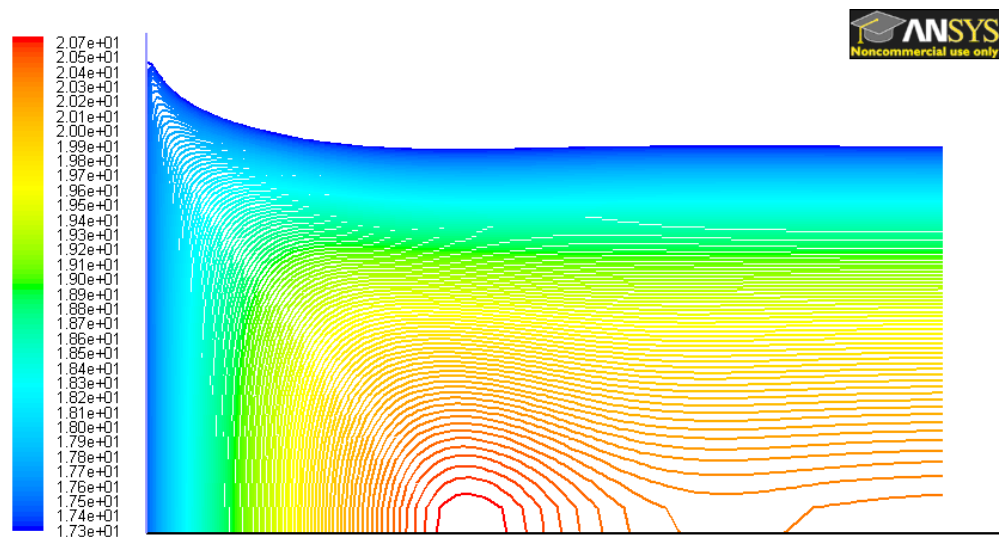

Contours of Velocity Magnitude $(\mathrm{m} / \mathrm{s})$

(b)

Figure 10: Comparison of contours of mean axial velocity: (a) measured data from Reichert [18], $\mathrm{u} / \mathrm{u}_{\mathrm{b}}$ (contour interval $\left.=0.02\right)$, (b) predicted data, $\mathrm{u}$, (contour interval $=0.10 \mathrm{~m} / \mathrm{s})$.

compared to the measured data. This can be attributed to the different inlet conditions employed in the experiments and simulations. In the simulations, a uniform velocity profile is used at the inlet while in the experiments, inlet boundary condition can be different from the simulations. The measurements show the waviness of the development of centerline velocity as shown in Fig. 9(a). It is notable that the simulations can capture the same physical phenomena even though there are some deviations between the magnitudes of 
the velocity contours. In general, the present predictions show a satisfactory agreement with the experimental data presented by Reichert [18].

\section{Conclusions}

In the present study, we report the results of a detailed and systematic numerical investigation of developing turbulent pipe flow. Two-dimensional, axisymmetric computational scheme has been devised for determining the flow development in the entrance region of a circular pipe at different Reynolds numbers. Simulations are performed using commercial CFD software ANSYS FLUENT 12.0.

The present results indicate that the development of the mean velocity field of turbulent pipe flow is non-asymptotic. However, developing laminar flow shows an asymptotic behavior. Regions of overshoot and undershoot of axial velocity profiles are discernible in the developing region. In association with this mean velocity behavior, a non-asymptotic development has been found for the wall shear stress. It has been suggested that the flow development may take place in a damped oscillatory manner such that even after 60 diameters downstream some flow adjustment is still occurring.

Contrary to Reichert [18] observation, the present results show that the higher the flow Reynolds number is, the further downstream the centerline mean velocity peak overshoot position moves. The reason for this is unknown at present and more detailed study is required. However, the present predictions show a good agreement with results presented by Wang [4]. In addition, the behavior of the development of integral boundary layers supports the present argument. As the Reynolds number increases, the magnitude of the centerline velocity overshoot reduces. The predictions show satisfactory agreement with the experimental data presented by Reichert [18]. It is notable that the simulations can capture the complex oscillatory behavior of the turbulent flow development.

\section{References}

[1] Holdhusen, J.S., 1952. Ph.D. Thesis, University of Minnesota, Minneapolis, Minnesota, 1952.

[2] Bowlus, D.A. \& Brighton, J.A., Am. Soc. Mech. Eng. Trans. 90, D, 431, 1968.

[3] Azad, R.S. \& Hummel, R.H., Can. J. Phys., 49, 2917, 1971.

[4] Wang, Y.Q., Prediction of developing turbulent pipe flow by a modified k$\varepsilon-\gamma$ model, Ph.D. Thesis, The University of Manitoba, 1999.

[5] Klein, A., Review: Turbulent developing pipe flow, Journal of Fluids Engineering, 103, pp. 243-249, 1981.

[6] Schlichting, H., Boundary layer theory, $6^{\text {th }}$ edition, McGraw Hill, 1968.

[7] Barbin, A.R. \& Jones, J.B., Turbulent flow in the inlet region of a smooth pipe, Journal of Basic Engineering, pp. 29-34, 1963. 
[8] Cockrell, D.J., Effect of inlet and outlet conditions on pipe and duct components, The $9^{\text {th }}$ Members Conference, Cranfield, SP 929, The British Hydrodynamics Research Association, 1967.

[9] Weir, J., Priest, A.J. \& Sharan, V.K., Research note: The effect of inlet disturbances on turbulent pipe flow, Journal of Mechanical Engineering Science, 16(3), pp. 211-213, 1974.

[10] Deissler, R.G., analytical and experimental investigation of adiabatic turbulent flow in smooth tubes, NACA T.N.2138, 1950.

[11] Sale, D.E., Entrance effects in incompressible diffuser flow, M.Sc. Thesis, University of Manchester, 1967.

[12] Reichert, J.K. \& Azad, R.S., Nonasymptotic behaviour of developing turbulent flow, Can. J. Phys., 54, pp. 268-278, 1976.

[13] Kays, W., Crawford, M., \& Weigand, B., Convective heat and mass transfer, $4^{\text {th }}$ edition, McGraw-Hill, 2005.

[14] Sovran, G. \& Klomp, E., Experimentally determined optimum geometries for rectilinear diffusers with rectangular, conical or annular cross-section, Fluids mechanics of internal flow, Elsevier Publishing Company, Amsterdam, 1967.

[15] FLUENT 12.0, User Manual, 2009.

[16] Durst, F., Ray, S., Unsal, B. \& Bayoumi, O. A., The Development Lengths of Laminar Pipe and Channel Flows, J. Fluids Eng., 127, pp. 1154-1160, 2005.

[17] Bradshaw, P., Review - Complex turbulent flows, ASME Journal of Fluid Engineering, 97(2), pp. 146-154.

[18] Reichert, J.K., A study of developing turbulent flow, PhD Thesis, The University of Manitoba, 1977.

[19] Chien, K. Y., Predictions of channel and boundary layer flows with a lowReynolds-number turbulent Model, AIAA Journal, 20(1), pp. 33-38, 1982. 